\title{
Pulmonary Manifestations of Collagen Vascular Diseases
}

\author{
C. P. Dokwal
}

The collagen vascular diseases (CVDs) include a heterogeneous group of chronic inflammatory immunologically-mediated systemic diseases, such as rheumatoid arthritis (RA), systemic lupus erythematosus (SLE), systemic sclerosis (SSc), Sjogren's syndrome (SS), polymyositis (PM)/dermatomyositis (DM), and mixed connective tissue disease (MCTD). They present with a wide range of clinical manifestations. The clinical features in CVDs frequently overlap causing much clinical confusion.

The lung is frequently affected in CVDs and is the cause of significant morbidity and mortality. The common pulmonary manifestations include pleural disease, pulmonary fibrosis, bronchiolitis obliterans, obliterans, organizing pneumonia, bronchiectasis, aspiration pneumonia, and diaphragmatic weakness. In the following paragraphs, the pulmonary manifestations in various collagen vascular diseases are discussed.

\section{SYSTEMIC LUPUS ERYTHEMATOSUS}

Systemic lupus erytehmatosus (SLE) is a multisystem autoimmune disease which shows a great variability in its presentation and course. Most patients have at least four of the American College of Rheumatology Criteria for SLE.1 Patients frequently present with systemic constitutional symptoms, such as fever, malaise, weight loss, fatigue, lymphadenopathy and hepatosplenomegaly, and these manifestations are seen throughout the course of SLE. Skin, musculoskeletal, lung, and renal systems are also commonly involved in SLE.

The pleuro-pulmonary involvement occurs in about 50-60\% of patients, especially later in the course of the disease when other organs are already affected. Pleuritis and pleural effusion are the most common pulmonary manifestation, although, lung parenchymal disease, pulmonary vascular disease, upper airway involvement, and diaphragmatic dysfunction also occur.

\section{Pleuritis and pleural effusion}

The pleural effusion is seen in $50 \%$ of patients with SLE during the course of their illness, and is the presenting feature in $5-10 \% .2,3$ The autopsy series have revealed pleural involvement in up to two thirds of patients.4 Plural effusion is typically bilateral, often associated with pericarditis, and frequently recurs. Pleural fluid is exudative in nature with either lymphocytic or polymorphonuclear cell predominance. Small pleural effusion usually resolves spontaneously, but severe and persistent one requires drug therapy.

\section{Interstitial lung disease}

Interstitial lung disease (ILD) has been described in $4 \%$ of patients with long-standing SLE in the past 5, however recent HRCT series reveal that about $1 / 3$ rd of patients with SLE have ILD, most having early sub clinical disease. 6 It usually develops insidiously and is associated with recurrent pleural effusions.

Chest radiographs typically show diffuse alveolar opacities. HRCT of chest often reveals a cellular, fibrotic, or mixed nonspecific interstitial pneumonia (NSIP) pattern.7 Usual interstitial pneumonia (UIP) and lymphoid pneumonia (LIP), particularly in those with associated secondary Sjogren's syndrome, have also been described.7, 8 Rarely, organizing pneumonia has also been reported.

\section{Diffuse Alveolar Haemorrhage}

Diffuse alveolar haemorrhage (DAH) is a rare complication seen in $4 \%$ of patients with SLE. Pulmonary capillaritis is often the underlying cause in most patients with DAH.9 It is often fatal in $50 \%$ of cases despite therapy, although mortality is declining with aggressive treatment with corticosteroids and cyclophosphamide.9, 10 Glomerulonephritsi is also present in most patients with DAH.9 DAH should be differentiated from acute lupus pneumonitis which also presents similarly, however, significant systemic symptoms suggestive of a SLE flare are typically absent in DAH.11 DAH may also occur secondary to uraemia or thrombocytopenia.

Chest radiograph in DAH commonly reveals bilateral, often extensive, airspace consolidation. Histopathology examination of lung tissue reveals a non-specific alveolitis, although in some patients the infiltration of small arteries, arterioles, and capillaries by polymorphonuclear cells may be seen.

\section{Acute Lupus Pneumonitis}

Acute lupus pneumonitis (ALP) has been described in $1 \%$ to $12 \%$ of patients, and often has a serious outcome 2,12 , it often presents acutely with fever, cough, dyspnea, pleuritic pain, and hypoxia, and sometimes haemoptysis.13 Occasionally it may be the presenting feature of SLE .11 The chest roentgenogram typically shows bilateral areas of airspace consolidation particularly in lower lung zones, and is often associated with bilateral pleural effusions.

ALP is a diagnosis of exclusion. It should be differentiated from diffuse alveolar haemorrhage and infective pneumonias. Surgical biopsies often reveal a histology pattern consistent with diffuse alveolar damage with or without alveolar haemorrhage 14, 15 ALP requires therapy with high dosage of systemic corticosteroids, and in refractory cases with additional immunosuppressive therapy, such as cyclophosphamide. 


\section{Pulmonary Manifestations}

\section{Pulmonary vascular disease}

Acute reversible hypoxemia is extremely rare, and has been described in acutely ill patients. It presents clinically as a sudden onset of hypoxemia with normal radiologic findings, and often responds rapidly to corticosteroids. 16 Patients with this syndrome have diffusion abnormality which is believed to be due to occlusive vasculopathy as a result of neutrophil aggregation within pulmonary capillaries. 17

The prevalence of pulmonary arterial hypertension $(\mathrm{PAH})$ in SLE is about $14 \%$, however, it increases with the progression of the disease. 18 Raynaud's phemomenon is seen in $75 \%$ of SLE patients who also have PAH, compared with 20\%-30\% of those without clinically evident PAH. Although, $50 \%$ of SLE patients with $\mathrm{PAH}$ have been reported to have underlying vasculitis 19 , it has been found much less common in recent biopsy studies. The prognosis of patients with vasculitis is poor.

Antiphospholipid antibodies are present in up to two thirds of patients with SLE 20,21, and it poses more than six fold risk of venous thrombo-embolism in this patient subgroup. 22

\section{RHEUMATOID ARTHRITIS}

Rheumatoid arthritis (RA) is the most common autoimmune disease affecting $1-2 \%$ of general population.23, 24 The involvement of heart, skin, lung, and eye is seen in 50\% patients with RA.25, 26 Pulmonary complications are common and often occur within 5 years of initial diagnosis of RA. They are also the presenting manifestations in $10 \%$ of patients. The commonest pulmonary manifestations are pleuritis and interstitial lung disease (ILD).27 Lung involvement is second to infection as the most common cause of death in patients with RA (18\% vs $27 \%)$.

\section{Pleural disease}

It is seen clinically in about $3-5 \%$ of patients with RA, although post mortem studies reveal a prevalence of $40-80 \%$. It is mainly seen in patients who have already developed extra articular manifestations. Pleural thickening is more common than pleural effusions. Pleural effusions are usually unilateral and may be loculated. They commonly occur late in the course of the disease, and may be associated with pericarditis and subcutaneous nodules. They are usually asymptomatic, but tend to recur after aspiration. The Pleural fluid is exudative with high protein component; however its glucose content is characteristically very low. Occasionally, pleural fluid may appear chylous due to high cholesterol content. Small pleural effusions do not require any specific therapy, and usually resolve spontaneously, but large effusions require aspiration. The recurrent pleural effusion may be treated with corticosteroid or pleurodesis.

\section{Interstitial Lung Disease}

ILD is the commonest and most serious complication seen in $15-20 \%$ of patients with RA. The usual interstitial pneumonia
(UIP) is the commonest histological pattern followed by nonspecific interstitial pneumonia (NSIP). Cryptogenic organizing pneumonia (COP) is less frequent. ILD typically occurs a few years after the onset of joint symptoms, however, it may be the first manifestation in $15 \%$ to $20 \%$ of RA patients. Chest radiograph typically shows reticular or reticulo nodular opacities in the lower lung fields. HRCT shows a reticular pattern and irregular septal thickening, mainly in the peripheral and lower lung zones. Honeycombing is a late manifestation of ILD. Rarely, some patients present with progressive upper lobe fibrosis and cavitations.

\section{Pulmonary Nodules}

Pulmonary nodules occur in up to $1 \%$ of patients. They are usually associated with advanced disease and subcutaneous nodules. They may be single or multiple, varying from $1-3 \mathrm{~cm}$ in diameter. They tend to be subpleural, often cavitate leaving thick-walled cavities. Occasionally, such cavities may be thin walled, which may disappear gradually. Rarely, rheumatoid nodules are seen in patients without RA, although some patients have developed RA disease later. Pulmonary nodules are rare in coal worker's pneumoconiosis, however, they may cause progressive massive fibrosis in some patients.

Bronchiolitis Obliterans/Bronchiolitis Obliternas Organizing Pneumonia (BOOP)

Occasionally, a severe rapidly progressive form of airway obstruction develops in patients with RA who present with widespread inspiratory crackles and often a mid inspiratory squeak. Chest radiograph is usually normal, but may show over inflation. HRCT of chest may show a characteristic mosaic pattern of attenuation and perfusion due to redistribution of blood flow away from the areas of abnormal ventilation. In early cases, the end-expiratory HRCT scan may show air-trapping when end-inspiratory scan is normal. Lung function studies show a severe obstructive deficit with low transfer factor for carbon monoxide (TLco). The autopsy studies reveal a widespread fibrous obliteration of bronchioles. Corticosteroid is not effective and most patients die in 2-3 yrs, although some patients have survived up to a decade.

\section{Other Pulmonary Manifestations in RA}

Pulmonary infections are frequent in RA patients. Rarely, eosinophilic pneumonia, bronchocentric granulomatosis, pulmonary hypertension, and progressive loss of lung volume due to diaphragmatic dysfunction may also occur. Small airway disease has been described in up to $30 \%$ of patients. Bronchiectasis is also common. The involvement of crico-arytenoid joint of larynx may cause stridor, which in severe case may require tracheostomy.

\section{SYSTEMIC SCLEROSIS (SCLERODERMA)}

Systemic sclerosis (SSc) is a rare, chronic, multisystem disease of unknown cause that is characterized by fibrosis and 


\section{Pulmonary Manifestations}

vasculopathy of the skin and visceral organs. There are 2 forms of SSc, the limited SSc and the diffuse SSc disease. The limited SSc is seen in 70\% of patients, and it manifests with subcutaneous calcinosis, Raynaud's phenmomenon, esophageal dismobility, sclerodactily, and telangiectasis. It tends to involve the distal limbs and face and has a lower risk of internal-organ involvement. However, PAH is more common in limited SSc disease. About $70 \%$ of patients have circulating anticentromere antibodies. The diffuse form often involves both distal and proximal limbs, the face, trunk and internal organs and has an increased associated mortality rate as compared with the limited form. 28 Although patients without skin changes (systemic sclerosis sine scleroderma) do not meet established criteria for a diagnosis of systemic sclerosis, they may have many features of the disease.29, 30

The lung is commonly involved in SSc. Most patients with SSc have pulmonary symptoms, such as exertional dyspnea and dry cough. The Interstitial lung disease and pulmonary vascular disease are the most common pulmonary complications. Aspiration pneumonia is also frequent due to oesophageal dysfunction, however, pleural disease is relatively rare, and is usually accompanied by lung parenchymal involvement.

ILD is seen in $20-60 \%$ of patients. It can occur in both limited and diffuse SSc. It rarely precedes scleroderma. Chest radiograph typically shows a bibasilar fine reticular pattern in the initial stages which progresses to coarse reticulation and honeycombing.31 NSIP is the most common histologic pattern, followed by UIP.24, 32, 33

HRCT scan of chest is more sensitive in diagnosing early ILD. It typically reveals lower lobes and peripheral involvement with areas of ground-glass attenuation, reticular shadowing, and poorly defined sub pleural nodules. Honeycombing and traction bronchiectasis predominate in long standing cases.

\section{MIXED CONNECTIVE TISSUE DISEASE}

Mixed connective tissue disease (MTCD) is a syndrome characterized by overlapping features of SSc, SLE, and $\mathrm{PM} / \mathrm{DM}$. The presence of high titres of antibodies against U1 small nuclear ribonucleoprotein (RNP) is a must for the diagnosis. About $80 \%$ of patients with MTCD have respiratory system involvement.34 Many patients with MTCD present with Raynaud's phenomenon, swollen hands, sclerodactyly, arthritis, PM and pulmonary involvement. 35

The common lung manifestations include ILD, pleural effusion and pulmonary arterial hypertension. ILD occurs in up to $65 \%$ of patients. 36 It usually occurs within first 2 to 4 years of onset of the disease. Rarely, ILD may be the first or predominant manifestation of MTCD .37 NSIP is the most common histologic pattern on lung biopsy.24, 38

Other pulmonary disorders include pulmonary vasculitis, pulmonary thrombo embolism, aspiration pneumonia, alveolar haemorrhage, pulmonary nodules, cysts, mediastinal lymphadenopathy, and respiratory muscle dysfunction.

\section{SJOGREN'S SYNDROME}

Sjogren's syndrome (SS) is characterized by lymphocytic infiltration of exocrine glands and other numerous extra glandular sites with resultant sicca syndrome.39 It can occur alone as primary Sjogren syndrome (pSS) or in association with other autoimmune diseases, such as RA, SLE, and progressive SS known as secondary Sjogren's syndrome ( $\mathrm{SSS})$. It is predominantly seen in middle aged women with a female/male ratio of 9:1 .40 It has recently been proposed that, the diagnosis of SS should include four of the following features: ocular symptoms of inadequate tear production, corneal damage due to inadequate tear production, dryness of mouth due to inadequate salivary production, salivary gland histopatholgy showing presence of foci of lymphocytes, impaired salivary gland function on clinical testing, and the presence of antibodies (anti-SS-A/Ro or anti-SS-B/La, or both), however, the compatible histologic findings or presence of auto antibodies has to be one of the four criteria. 41

Lung involvement is common extra glandular manifestation of SS, and is seen in $9 \%$ to $75 \%$ patients. 42,43 However, a recent study reported lung involvement in $11 \%$ of patients with primary Sjogren's syndrome.44 The common pulmonary manifestations in SS include small airway disease, large airway obstruction or desiccation of the tracheobronchial tree, and interstitial lung disease.

\section{Airway involvement}

Upper airway is commonly involved in SS. The dryness of the oral and nasal mucosa affects smell and taste functions. Upper airway bleeding, sinusitis, and septal perforation may also occur. 45

Dry cough is frequently present due to dryness of mucosa of trachea (xerotrachea) and large airways. Bronchial glands in large airway reveal changes similar to those in salivary exocrine glands, and mucosa shows lymphocytic infiltration predominantly with CD4 T lymphocytes.

Small airways are commonly involved in SS, but severe symptoms due to this are rare. With the progression of the disease, air trapping, mosaic attenuation, and bronchiectasis may manifest on CT scan of chest.

\section{Follicular Bronchiolitis}

Follicular bronchiolitis is commonly associated with lymphocytic bronchitis, bronchiolitis, or LIP in patients with primary SS. Histological examination often reveals nodules of lymphocytic infiltrates with reactive germinal centres surrounding the terminal and respiratory bronchioles.

\section{Diffuse Interstitial Lung Disease (ILD)}




\section{Pulmonary Manifestations}

Diffuse ILD is seen in 5\% of patients with SS. Rarely, ILD may present without the sicca syndrome. NSIP is the commonest Interstitial lung disease in primary SS, which commonly manifests as increased reticular markings, ground-glass opacities, and traction bronchiectasis on $\mathrm{HRCt}$ scan of chest.46, 47, 48 Honeycombing is a late feature of NSIP. LIP is seen in $0.9 \%$ of patients with SS, whereas $25 \%$ of adults with LIP suffer from SS. A pattern of ground-glass opacities with thin-walled cysts are found in $50 \%$ of patients with LIP with other frequent radiologic findings being centrilobular nodules and interlobular septal thickening. Rarely, pleural effusion may also be present.49 UIP is rare in SS. HRCT of chest often reveals fibrosis, honeycombing, and traction bronhciectasis in lower lobes. Organizing pneumonia (OP) is extremely rare in SS with only a handful of cases reported. Recently, a patient with OP has been described, who subsequently developed SS 3 years later.50

\section{Lymphoma}

The risk of lymphoma is 44 times higher in Sjogren's syndrome than that in a healthy population.51 Lymphoma develops in $4-8 \%$ of patients with SS with NHL being the commonest type.52, 53 Primary pulmonary lymphoma is also seen in $1 \%-2 \%$ of patients which can present as a solitary or multifocal nodules, bilateral alveolar infiltrates, or interstitial markings. 43,54

\section{Other Manifestations}

Despite lung being commonly involved in SS, pulmonary hypertension is rare.55, 56 Pleural effusions and pleural thickening are also extremely rare conditions in SS.

\section{POLYMYOSITIS/DERMATOMYOSITIS}

Polymyositis (PM) and dermatomyositis (DM) affect skeletal muscles and various other organs. About $5 \%$ of patients with PM/DM develop lung manifestations with ILD and aspiration pneumonia being the commonest. Pulmonary vascular disease is rare. As $\mathrm{PM} / \mathrm{DM}$ also occurs in malignancy, an active search for malignancy should be made in those who present with PM/DM.

The most common radiographic finding is aspiration pneumonia due to pharyngeal muscle weakness. The diaphragmatic weakness leads to high diaphragmatic elevation, reduced lung volumes, and basilar atelectasis.

ILD is seen in $50 \%$ of patients with PM/DM.57, 58 ILD commonly follows the musculoskeletal involvement, but may precede these in 1/3rd of cases.59, 60 ILD may be the sole manifestation in $20-30 \%$ of patients with DM/PM. 59, 60 , and in $10 \% 20 \%$ patients with amyopathic form of DM.

The lung bases are predominantly involved in ILD. Chest radiography reveals a reticulo-nodular pattern or honeycombing. HRCT of chest may reveal predominant linear abnormalities and ground-glass attenuation. Airspace consolidation is also often present in the lower lung zones with a peri bronchial and sub pleural distribution usually due to organizing pneumonia which is more common in PM/DM compared to other collagen vascular diseases.61 Although NSIP is the most common histological pattern, COP (cryptogenic organizing pneumonia), diffuse alveolar damage (DAD) and UIP are also frequently found.59

\section{RELAPSING POLYCHONDRITIS}

Relapsing polychondritis (RP) is a rare disease characterized by recurrent episodes of cartilaginous inflammation with degeneration, loss of structure, and fibrosis throughout the body. The ears, nose, and airways are the most frequently affected organs in RP. About 1/3rd of patients will also suffer from another rheumatologic disorder, such as vasculitis, RA, Sjogren's syndrome, and ankylosingspondylitis. Rarely RP is seen in patients with inflammatory bowel disease, primary biliary cirrhosis, and myelodysplastic syndrome.

Airway manifestations are seen in $50 \%$ of patients with RP during the course of their illness. These are also the most serious complications presenting a diagnostic and therapeutic challenge.62 It may be the presenting feature in $10 \%$ of patients. The common airway involvement in RP includes subglottic stenosis, tracheal stenosis, tracheal wall thickening and calcification, and tracheobronchomalacia.

Respiratory symptoms are common in patients with RP, such as cough, dyspnea, hoarseness of voice, and stridor. Bronchoscopy is performed in patients having respiratory symptoms to assess for mucosal inflammation as a marker for active disease. The common bronchoscopic features are generalized redness of mucosa, mucosal oedema, tracheobronchomalacia with airway collapse, fixed narrowing and stenosis from granulation tissue and peribronchial fibrosis. However, tracheobronchomalacia and focal stenosis in the tracheobronchial tree are the commonest bronchoscopic findings.63 The sparing of posterior tracheal mucosa is an important diagnostic clue.

Most patients with respiratory symptoms reveal computed tomography (CT) scan abnormalities including tracheobronchomalacia and air trapping during expiratory scan which may not be apparent in inspiratory CT scan of the chest. Hence, the dynamic CT imaging is recommended in patients with suspected airway involvement.64 Recent developments in the imaging modalities such as threedimensional imaging will allow early and improved detection of airway involvement in RP.

\section{ANKYLOSING SPONDYLITIS}

Ankylosing spondylitis (AK) is a multisystem disorder of unknown pathogenesis which primarily affects the joints of the axial skeleton. It commonly affects young males, $90 \%$ of them being HLA-B27 positive. The progressive destruction of the joints results in stiffness of spine, chest, and pelvis.

The lung is involved in up to $30 \%$ of patients with chest wall restriction and upper lobe fibrosis being the commonest.6, 65 


\section{Pulmonary Manifestations}

The restrictive ventilator defect may develop as a result of either fusion of costovertebral joints and ankylosis of thoracic spine or anterior chest wall involvement.

The lung parenchymal involvement generally occurs many years after the onset of arthritic changes. Upper lobe fibrobullous disease is the most common lung manifestation described in $1.3 \%$ to $30 \%$ of patients with ankylosing spondylitis.66, 67 Bilateral apical scarring is believed to be the earliest pulmonary manifestation (54).67 HRCT of chest may reveal peripheral interstitial lung disease, apical fibrosis, bronchiectasis, paraseptal emphysema, tracheobronchomegaly. Cavitation is uncommon, and was reported in only 0.45 of patients in one series. 68 There is a higher risk of pneumothorax in patients with $\mathrm{AK}$, and it may be recurrent in some.

\section{CONCLUSIONS}

Patients with CVDs present with a wide range of pulmonary manifestations which cause a significant morbidity and mortality in this patient population. In addition, the frequent use of immunosuppressive therapy often makes them vulnerable to a wide range of infections which may create a diagnostic dilemma in many patients. To make the matter worse, some patients may present with lung involvement as a sole or predominant feature of an underlying VCD. Thus, a presence of a CVD should be suspected in a proper clinical context, particularly in patients with interstitial lung diseases, so that unnecessary delay in arriving at a diagnosis is avoided, and outcome in this patient group is improved.

\section{References:}

1. Tan EM, Fries JF, Masi AT. The 1982 revised criteria for the classification of systemic lupus erythematosus. Arthritis Rheum. 1982;25:1271-1272.

2. Wiedemann HP, Matthay RA. Pulmonary manifestations of systemic lupus erythematosus. J Thorac Imaging. 1992;7:1-18.

3. Pines A., Kaplinsky N., Olchovsky D. Pleuro-pulmonary manifestations of systemic lupus erythematosus: clinical features of its subgroups. Prognostic and therapeutic implications. Chest. 1985;88:129-135.

4. Purnell DC, Baggenstoss AH, Olsen AM. Pulmonary lesions in disseminated lupus erythematosus. Ann Intern Med. 1955;42:619-628.

5. Haupt HM, Moore GW, Hutchins GM. The lung in systemic lupus erythematosus: analysis of the pathologic changes in 120 patients. Am J Med. 1981;71:791-798.

6. Fenlon HM, Casserley I, Sant SM, Breatnach E. Plain radiographs and thoracic high-resolution CT in patients with ankylosing spondylitis. AJR Am J Roentgenol. 1997;168:10671072.

7. Tansey D, Wells AU, Colby TV. Variations in histological patterns of interstitial pneumonia between connective tissue disorders and their relationship to prognosis. Histopathology. 2004;44:585-596.

8. Schattner A, Aviel-Ronen S, Mark EJ. Accelerated usual interstitial pneumonitis, anti-DNA antibodies and hypocomplementemia. J Intern Med. 2003;254:193-196.

9. Zamora MR, Warner ML, Tuder R, Schwarz MI. Diffuse alveolar hemorrhage and systemic lupus erythematosus. Clinical presentation, histology, survival and outcome. Medicine.1997;76(3):192-202.

10. Santos-Ocampo AS, Mandell BF, Fessler BJ. Alveolar hemorrhage in systemic lupus erythematosus: presentation and management. Chest. 2000;118(4):1083-1090.

11. Matthay RA, Schwarz MI, Petty L. Pulmonary manifestatiosn of systemic lupus erythematosus; review of twelve cases of acute lupus pneumonitis. Medicine. 1975; 54(5):397-409.

12. Cheema GS, QuismorioFP Jr. Interstitial lung disease in systemic lupus erythematosus. Curr Opin Pulm Med. 2000;6:424-429.

13. Boulware DW, Hedgpeth MT. Lupus pneumonitis and anti-SSA(Ro) antibodies. J Rheumatol.1989;16:479-481.

14. Keane MP, Lynch JP. Pleuropulmonary manifestations of systemic lupus erythematosus. Thorax 2000;55:159-166.

15. Harvey AM, Shulman LE, Tumulty PA. Systemic lupus erythematosus: review of the literature and clinical analysis of 138 cases. Medicine. $1954 ; 33,291-437$.

16. Abramson SB, Dobro J, Eberle MA. Acute reversible hypoxemia in systemic lupus erythematosus. Ann Intern Med. 1991;114:941947.

17. Belmont HM, Buyon J, Giorno R, Abramson S. Up-regulation of endothelial cell adhesion molecules characterizes disease activity in systemic lupus erythematosus: the Schwartzman phenomenon revisited. Arthritis Rheum. 1994;37:376383

18. Winslow TM, Ossipov MA, Fazio GP. Five-year follow-up study of the prevalence and progression of pulmonary hypertension in systemic lupus erythematosus. Am Heart J. 1995; 129:510515.

19. Fayemi AO. Pulmonary vascular disease in systemic lupus erythematosus. Am J Clin Pathol. 1976;65:284290.

20. Somers E, Magder LS, Petri M. Antiphospholipid antibodies and incidence of venous thrombosis in a cohort of patients with systemic lupus erythematosus. J Rheumatol .2002;29:25312536.

21. Ruiz-Irastorza G, Egurbide MV, Ugalde J. High impact of antiphospholipid syndrome on irreversible organ damage and survival of patients with systemic lupus erythematosus. Arch Intern Med. 2004; 164:7782.

22. Wahl DG, Guillemin F, de Maistre E. Risk for venous thrombosis related to antiphospholipid antibodies in systemic lupus erythematosus: a metaanalysis. Lupus. 1997;6:467473.

23. Crestani B. The respiratory system in connective tissue disorders Allergy. 2005; 60:715734

24. Kim DS, Yoo B, Lee JS. The major histopathologic pattern of pulmonary fibrosis in scleroderma is nonspecific interstitial pneumonia. Sarcoidosis Vasc Diffuse Lung Dis. 2002;19:121127.

25. Horton MR. Rheumatoid arthritis associated interstitial lung disease. Crit Rev Comput Tomogr. 2004;45:429440.

26. Maradit-Kremers H, Nicola PJ, Crowson CS, Ballman KV, Gabriel SE. Cardiovascular death in rheumatoid arthritis: a population-based study. Arthritis Rheum. 2005;52:722732.

27. American Thoracic Society/European Respiratory Society. International Multidisciplinary Consensus Classification of the Idiopathic Interstitial Pneumonias. Am J Respir Crit Care Med. 2002;165:277304

28. Bielsa I, Ariza A. Deep morphea. Semin Cutan Med Surg. 2007;26:90 95

29. Lomeo RM, Cornella RJ, Schabel SI, Silver RM. Progressive systemic sclerosis sine scleroderma presenting as pulmonary interstitial fibrosis. Am J Med. 1989;87:525-527.

30. Poormoghim H, Lucas M, Fertig N, Medsger TA. Systemic sclerosis sine scleroderma: demographic, clinical, and serologic features and survival in forty-eight patients. Arthritis Rheum. 2000;43:444-451.

31. Arroliga AC, Podell DN, Matthay RA. Pulmonary Manifestations of scleroderma. J Thorac Imaging. 1990;7:30-45.

32. Bouros D, Wells AU, Nicholson AG. Histopathologic subsets of fibrosing alveolitis in patients with systemic sclerosis and their 


\section{Pulmonary Manifestations}

relationship to outcome. Am J Respir Crit Care Med. 2002; $165: 15811586$.

33. Fujita J, Yoshinouchi T, Ohtsuki Y. Non-specific interstitial pneumonia as pulmonary involvement of systemic sclerosis. Ann Rheum Dis. 2001;60:281283.

34. Sharp GC, Irvin WS, Tan EM, Gould RG, Holman HR. Mixed connective tissue diseasean apparently distinct rheumatic disease syndrome associated with a specific antibody to an extractable nuclear antigen (ENA). Am J Med. 1972;52:148159.

35. Venables PJ. Mixed connective tissue disease. Lupus. 2006;15:132137.

36. Prakash UB. Respiratory complications in mixed connective tissue disease. Clin Chest Med. 1998;19:733-746.

37. Saito Y, Terada M, TakadaT. Pulmonary involvement in mixed connective tissue disease: comparison with other collagen vascular disease using high-resolution CT. J Comput Assist Tomogr. 2002;26:349 $-357$.

38. Park JH, Kim DS, Park IN. Prognosis of fibrotic interstitial pneumonia: idiopathic versus collagen vascular disease-related subtypes. Am J Respir Crit Care Med. 2007; 175:705-711.

39. Bloch KJ, Buchanan WW, Wohl MJ, Bunim JJ. Sj gren's syndrome: a clinical, pathological, and serological study of sixty-two cases. Medicine. 1965;44:187231

40. Mavragani CP, Moutsopoulos HM. The geoepidemiology of Sjogren's syndrome. Autoimmun Rev. 2010;9:A305-A310.

41. Vitali C, Bombardieri S, Jonsson R. Classification criteria for Sjhogren's syndrome: a revised version of the European criteria proposed by the American-European Consensus Group. Ann Rheum Dis. 2002;61:554558.

42. Constantopoulos SH, Papadimitriou CS, Moutsopoulos H.M. Respiratory manifestations in primary Sjogren's syndrome. A clinical, functional, and histologic study. Chest. 1985:88:226-229.

43. Strimlan CV, Rosenow EC, Divertie MB. Pulmonary manifestations of Sjogren's syndrome. Chest. 1976;70:354-361.

44. Yazisiz V, Arslan G, Ozbudak IH. Lung involvement in patients with primary Sjogren's syndrome: what are the predictors? Ann Rheum Dis. 2002;61(6):554-558.

45. Freeman SR, Sheehan PZ, Thorpe MA. Ear, nose, and throat manifestations of Sjogren's syndrome: retrospective review of a multidisciplinary clinic. J Otolaryngol. 2005;34:20-24.

46. Ito I, Nagai S, Kitaichi M. Pulmonary manifestations of primary Sjogren's syndrome: a clinical, radiologic, and pathologic study. Am J Respir Crit Care Med. 2005; 171:632-638.

47. Kim EA, Lee KS, Johkoh T. Interstitial lung diseases associated with collagen vascular diseases: Radiologic and histopathologic findings. Radiographics. 2002;22:s151-s165.

48. Parambil JG, Myers JL, Lindell RM. Interstitial lung disease in primary Sjogren syndrome. Chest. 2006;130:1489-1495.

49. Johkoh T, Muller NL, Pickford HA. Lymphocytic interstitial pneumonia: thin-section $\mathrm{CT}$ findings in 22 patients. Radiology. 1999;212:567-572.

50. Henriet AC, Diot E, Marchand-Adam S. Organising pneumonia can be the inaugural manifestation in connective tissue diseases, including Sj gren's syndrome. Eur Respir Rev. 2010; 19:116,161-163.

51. Kassan SS, Thomas TL., Moutsopoulos HM. Increased risk of lymphoma in sicca syndrome. Ann Intern Med. 1978;89:888-892.

52. Tonami H., Matoba M, Kuginuki Y. Clinical and imaging findings of lymphoma in patients with Sjogren syndrome. J Comput Assist Tomogr. 2003;27:517-524.

53. Voulgarelis M, Dafni UG, Isenberg DA. Malignant lymphoma in primary Sjogren's syndrome: a multicenter, retrospective, clinical study by the European Concerted Action on Sjogren's syndrome. Arthritis Rheum. 1999;42:1765-1772.

54. Franquet T, Gimenez A, Monill JM. Primary Sjogren's syndrome and associated lung disease: CT findings in 50 patients. Am J Roentgenol. 1997; 169:655-658.

55. Bertoni M, Niccoli L, Porciello G. Pulmonary hypertension in primary Sjogren's syndrome: report of a case and review of the literature. Clin Rheumatol. 2005; 24:431-434.

56. Hedgpeth M.T, Boulware DW. Pulmonary hypertension in primary Sjogren's syndrome. Ann Rheum Dis. 1988;47:251-253.

57. Fathi M, Dastmalchi M, Rasmussen E. Interstitial lung disease, a common manifestation of newly diagnosed polymyositis and dermatomyositis. Ann Rheum Dis. 2004;63(3):297-301.

58. Selva-O'Callaghan A., Labrador-Horrillo M., Munoz-Gall X Polymyositis/dermatomyositis-associated lung disease: analysis of a series of 81 patients. Lupus. 2005;14(7):534-542.

59. D o u g l a s W W, T a ze la a r H D, H a r tm a n T E Polymyositisdermatomyositis-associated interstitial lung disease. Am J Respir Crit Care Med. 2001;164:11821185.

60. Marie I, Hachulla E, Cherin P. Interstitial lung disease in polymyositis and dermatomyositis. Arthritis Rheum. 2002;47:614662.

61. Ikezoe J, Johkoh T, Kohno N. High-resolution CT findings of lung disease in patients with polymyositis and dermatomyositis. J Thorac Imaging. 1996;11.(4):250-259.

62. Eng J, Sabanathan S. Airway complications in relapsing polychondritis. Ann Thorac Surg. 1991;51:686-692.

63. Ernst A, Rafeq S, Boiselle P. Relapsing polychondritis and airway involvement. Chest 2009;135:1024-1030.

64. Lee KS, Ernst A, Trentham D. Prevalence of functional airway abnormalities in relapsing polychondritis. Radiology. 2006;240:565573.

65. Rumancik WM, Firooznia H, Davis MS Jr. Fibrobullous disease of the upper lobes: an extraskeletal manifestation of ankylosing spondylitis. J Comput Tomogr. 1984; 8:225229.

66. Kentala E., Repo UK, Lehtipuu AL. HLA-antigens and pulmonary upper lobe fibrocystic changes with and without ankylosing spondylitis. Scand J Respir Dis. 1978;59:8-12.

67. Rosenow EC, Strimlan CV, Muhm JR. Pleuropulmonary manifestations of ankylosing spondylitis. Mayo Clin Proc. 1977;52:641-49.

68. Crompton GK, Cameron SJ, Langlands AO. Pulmonary fibrosis, pulmonary tuberculosis, and ankylosing spondylitis. Br J Dis Chest. 1974:68:5156.

69. Preliminary criteria for the classification of systemic sclerosis (scleroderma). Subcommittee for scleroderma criteria of the American Rheumatism Association diagnostic and therapeutic criteria committee. Arthritis Rheum. 1980;23(5):581-590.

70. Mayberry P, Primack SL, Muller NL. Thoracic manifestations of systemic autoimmune diseases: radiographic and high-resolution CT findings. Radiographics 2000;20:1623-1635.

71. Matthay RA, Schwarz MI, Petty L. Pulmonary manifestations of systemic lupus erythematosus; review of twelve cases of acute lupus pneumonitis. Medicine (Baltimore). 1975;54(5):397-409.

72. Kim DS. Interstitial lung disease in rheumatoid arthritis: recent advances. Curr Opin Pulm Med. 2006;12:346353.

73. Kanathur N, Lee-Chiong T. Pulmonary manifestations of ankylosing spondylitis. Clin Chest Med. 2010;31:547-554.

74. Cain HC, Noble PW, Matthay RA. Pulmonary manifestations of Sjogren's syndrome. Clin chest Med. 1998;19:687-699. 